\title{
Experimental verification of photostability for free- and bound-amino acids exposed to $\gamma$-rays and UV irradiation
}

\author{
Yoshinori Takano $^{1}$, Takeo Kaneko ${ }^{2}$, Kensei Kobayashi $^{2}$, Daisuke Hiroishi ${ }^{3}$, Hidematsu Ikeda ${ }^{3}$, and Katsumi Marumo ${ }^{1}$ \\ ${ }^{1}$ Institute for Marine Resources and Environment (MRE), National Institute for Advanced Industrial Science and Technology (AIST), \\ AIST Central 7, 1-1-1 Higashi, Tsukuba 305-8567, Japan \\ ${ }^{2}$ Department of Chemistry and Biotechnology, Yokohama National University, \\ 79-5 Tokiwadai, Hodogaya-ku, Yokohama 240-8501, Japan \\ ${ }^{3}$ Research Center for Nuclear Science and Technology, University of Tokyo, \\ 2-11-16 Yayoi, Bunkyo-ku, Tokyo 113-0032, Japan
}

(Received December 14, 2003; Revised June 7, 2004; Accepted June 13, 2004)

\begin{abstract}
The photo-stability of simulated free and bound amino acids exposed to photon sources of $1.2-1.3 \mathrm{MeV} \gamma$ rays from a ${ }^{60} \mathrm{C}^{\circ}$ source and ultra-violet (UV) irradiation from a $10 \mathrm{eV}$ deuterium lamp was examined. The free amino acids in aqueous solution were drastically decreased while the bound amino acids in aqueous solution were slightly decreased with the duration of $\gamma$-rays and UV irradiation. The apparent half-lives $\left(t_{1 / 2}\right)$ of bound serine and threonine under $\gamma$-rays irradiation were more than 10.6 and 27.9 times as stable as the free amino acids, respectively. The photo-stability of bound amino acids was greater than that of free amino acids under irradiation by high-energy photons. These results strongly suggest that bound, but not free, amino acids could be found in extraterrestrial environments. Radio- or photo-alteration processes are mainly decomposition pathways, with the production of secondary amino acids via decomposition of the $\alpha$-carboxylic group; $\beta$-alanine and $\gamma$-aminobutyric acids, $\alpha$-decarboxylation products of aspartic acid and glutamic acid, respectively, were detected.
\end{abstract}

Key words: Photostability, free- and bound-amino acids, irradiation experiment, half-lives.

\section{Introduction}

In extraterrestrial environments, corpuscular beams such as protons or electrons and photon beams such as $\gamma$-rays or ultraviolet (UV) are universally present, and these energy sources facilitate the formation of interstellar or interplanetary organic compounds from cosmic media (Kobayashi et al., 2001). In fact, steady progress has been made in the identification of organic compounds in extraterrestrial environments by telescopic observation (Kuan et al., 2003, 2004) and through the examination of meteorites (Cronin et al., 1988). Telescopic observation of astrobiologically interesting organic compounds in extraterrestrial environment were systematically reviewed by Ehrenfreund and Charnley (2000): gas phase and grain surface formation pathways to organic molecules in dense interstellar clouds, using recent observations with the Infrared Space Observatory (ISO) and ground-based radio-telescopes. The main spectroscopic evidence for carbonaceous compounds in the diffuse interstellar medium is discussed regarding UV bump at $2200 \AA$, diffuse interstellar bands, extended red emission, and infrared absorption and emission bands. The evolution, survival, transport, and transformation of organics is monitored, from molecular clouds and the diffuse interstellar medium to their incorporation into solar system material such as comets and meteorites (Ehrendfreund and Charnley, 2000).

Carbonaceous chondrites, in particular, carry the abiotic

Copy right(c) The Society of Geomagnetism and Earth, Planetary and Space Science (SGEPSS); The Seismological Society of Japan; The Volcanological Society of Japan; The Geodetic Society of Japan; The Japanese Society for Planetary Sciences; TERRAPUB. record of proto-solar systems and primitive chemical evolution (Cronin et al., 1988; Cottin et al., 1999). Successful detection of enantiomeric excess of amino acids in the Murchison and Murray meteorites leads to a persuasive scenario for the exogenous origins of homochirality (Cronin and Pizzarello, 1997; Pizzarello and Cronin, 2000).

Since the pioneering laboratory experiment by Miller (1953), numerous kinds of simulation experiments for the study of prebiotic formation of organic compounds have been performed. Among these, Kobayashi et al. (1995, 1998) showed that a wide variety of organic compounds can be formed from mildly reduced gas mixtures by introducing high energy particles and that the yield of the products depends on the energy deposit. Recently, the prebiotic formation of amino acids at the extreme low temperature of 10 $\mathrm{K}$ was examined on an ice film (Briggs et al., 1992; Bernstein et al., 2002; Munoz Caro et al., 2002). The relationship between the formation of amino acid precursors and the energetics of nitrogen fixation was also evaluated in a simulated ice mantle of interstellar dusts in molecular clouds at $10 \mathrm{~K}$ (Takano et al., 2003). Kasamatsu et al. (1997) showed that amino acid precursors (molecules which are hydrolyzed to amino acids) could be formed by irradiating an icy mixture of carbon monoxide, ammonia and water with high energy protons. The energy yields (G-value rate, the number of formed molecule/100 eV of glycine) with $\gamma$-rays and UV irradiation were found to be independent of the starting material phase (Takano et al., 2002; Kobayashi et al., 2004).

Amino acids are the most familiar organic compounds since they are essential for the generation of life and 


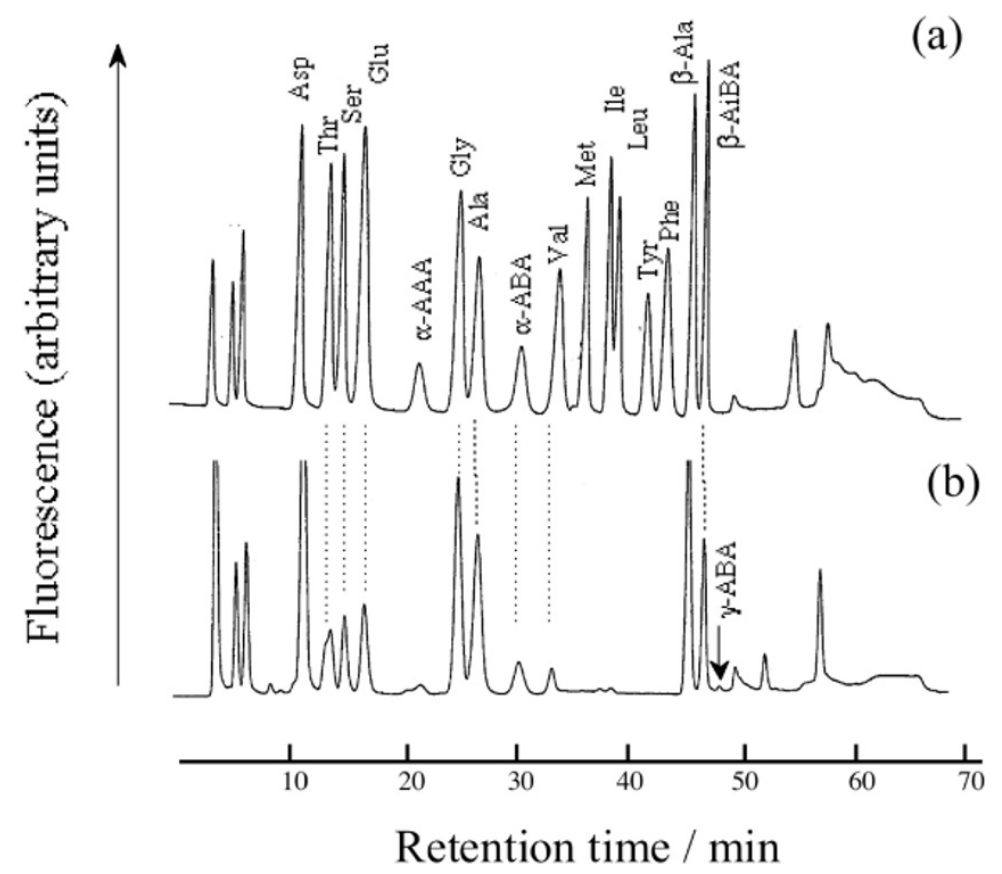

Fig. 1. Ion-exchange chromatograms of free amino acid analogs by $\gamma$-rays irradiation. (a) Irradiation for 0 hr, (b) irradiation for 3 hr. Abbreviations: Asp: aspartic acid, Thr: Threonine, Ser: serine, Glu: glutamic acid, $\alpha$-AAA: $\alpha$-aminoadipic acid, Gly: glycine, Ala: alanine, $\alpha$-ABA: $\alpha$-aminobutyric acid, Val: valine, Met: Methionine, Ile: isoleucine, Leu: leucine, Tyr: tyrosine, Phe: phenylalanine, $\beta$-Ala: $\beta$-alanine, $\beta$-AiBA: $\beta$-aminoisobutyric acid, $\gamma$-ABA: $\gamma$-aminobutyric acid.

the terrestrial biosphere. Primary products in irradiation experiments of chemical evolution are not free amino acids (monomer constituents), but amino acids precursors (Kobayashi et al., 1995, 1998). Prebiotically synthesized amino acids and nucleic acid bases (Kobayashi and Tsuji, 1997; Miyakawa et al., 2002) are detected in chromatograms after acid hydrolysis. However, only trace amounts of glycine could be detected among the experimental products prior to acid hydrolysis. Free amino acids might also be released from bound organic precursors in meteors by abiogenic processes such as hydro-alteration (e.g. Bernstein et al., 2002). Another difficulty in detecting free amino acid analogs in meteors is that they must remain intact despite bombardment with cosmic radiation such as $\gamma$-rays or UV irradiation while traveling to the Earth. Ehrenfreund et al. (2001) demonstrated laboratory studies testing the stability of amino acids against UV photolysis: two biological and two non-biological amino acids have been irradiated in frozen $\mathrm{Ar}, \mathrm{N}_{2}$, and $\mathrm{H}_{2} \mathrm{O}$ in order to simulate astrophysical conditions in the interstellar gas and on interstellar grains. The rate of photolysis is rather insensitive to the amino acid structure and to the ice matrix. However, UV photon does not penetrate ice mixtures up to $0.2 \mu \mathrm{m}$ for $\mathrm{N}_{2}$ and $\mathrm{H}_{2} \mathrm{O}$ ices (Ehrenfreund et al., 2001). Hence interior portion of organic compounds within icy mixtures will intact against UV photolysis. $\gamma$-rays irradiation experiment are useful for stability demonstration not only bounds compounds but also free one. Here we examine the stability of free and bound amino acids under irradiation with $\gamma$-rays and UV photons. Significant implications for the study of amino acid morphology in extraterrestrial environment were learned from comparisons of the photo-stability.

\section{Experimental Procedure}

Mixtures $\left(10^{-5} \mathrm{~mol} / \mathrm{l}\right)$ of free and bound amino acids in aqueous solution were made from a mixture of 16 free amino acid standards (AN-II; WAKO Pure Chemical Industries, Ltd., Japan) and human serum albumin (ca. 65,000 Da, WAKO Pure Chemical Industries, Ltd. Japan), respectively. Human serum albumin is one of globulin proteins, which is treated as a model of bound amino acids. Mixtures were prepared in de-ionized water that was further purified with a Millipore Milli-Q LaboSystemTM and Millipore Simpli Lab-UV (Japan Millipore Ltd.) to remove both inorganic ions and organic contaminants.

The free and bound amino acid aqueous mixtures were sealed in glass tubes under air after degassing by ultrasonication and irradiated with $\gamma$-rays $(1.2-1.3 \mathrm{MeV})$ at a dose rate of $7.15 \mathrm{~J} / \mathrm{h}$ from a ${ }^{60} \mathrm{Co}$ source at the Research Center for Nuclear Science and Technology, University of Tokyo. Dose rate, which was measured with a Fricke dosimeter (Ikeda and Suzuki, 1998). Then, the mixtures were subjected to UV photon (wavelength, over $160 \mathrm{~nm}$ ) under a $150 \mathrm{~W}$ deuterium lamp (L1835; Hamamatsu Photonics) equipped with a UV irradiation window and a synthesized quartz window at a dose rate of $2.05 \mathrm{~J} / \mathrm{h}$ for 24 hours (Takano et al., 2002, 2003; Kobayashi et al., 2004). Since irradiation window was attached with quartz window, above $160 \mathrm{~nm}$ wavelength was obtained. The irradiation experiments were performed at room temperature under ambient atmosphere. Blank runs were also performed in the same manner.

Since our photolysis experiments might produce amino acids and/or amino acid precursors, and this could change the apparent photo-stability, we looked for and were able to discern the survivability of amino acids using HPLC (see Fig. 3 

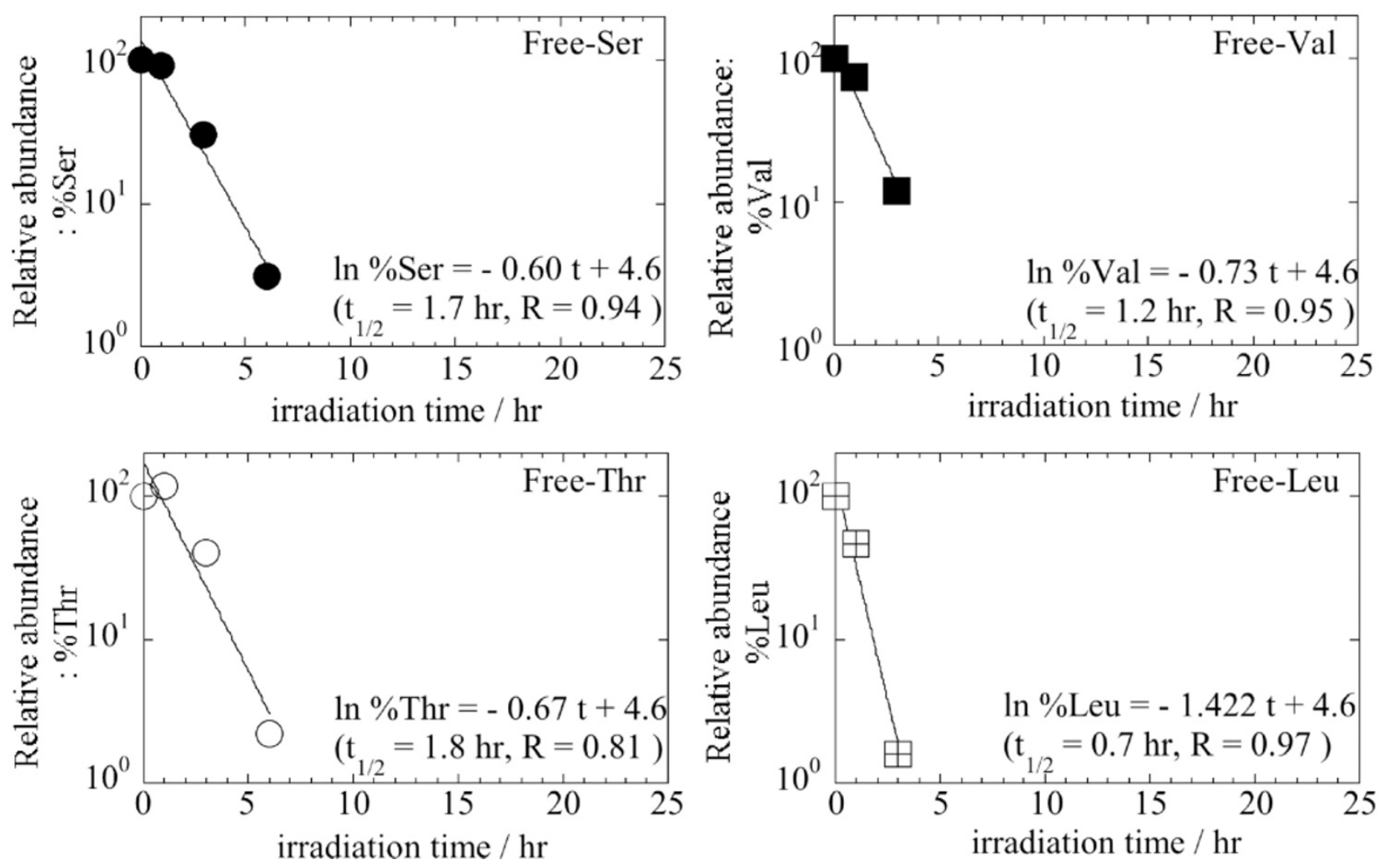

Fig. 2(a). Semi-logarithmic linear relations between relative abundance and radiation time to determine half-life of free amino acid analogs by $\gamma$-rays irradiation experiment. Free amino acids of serine, valine, threonine and leucine were shown as typical examples.
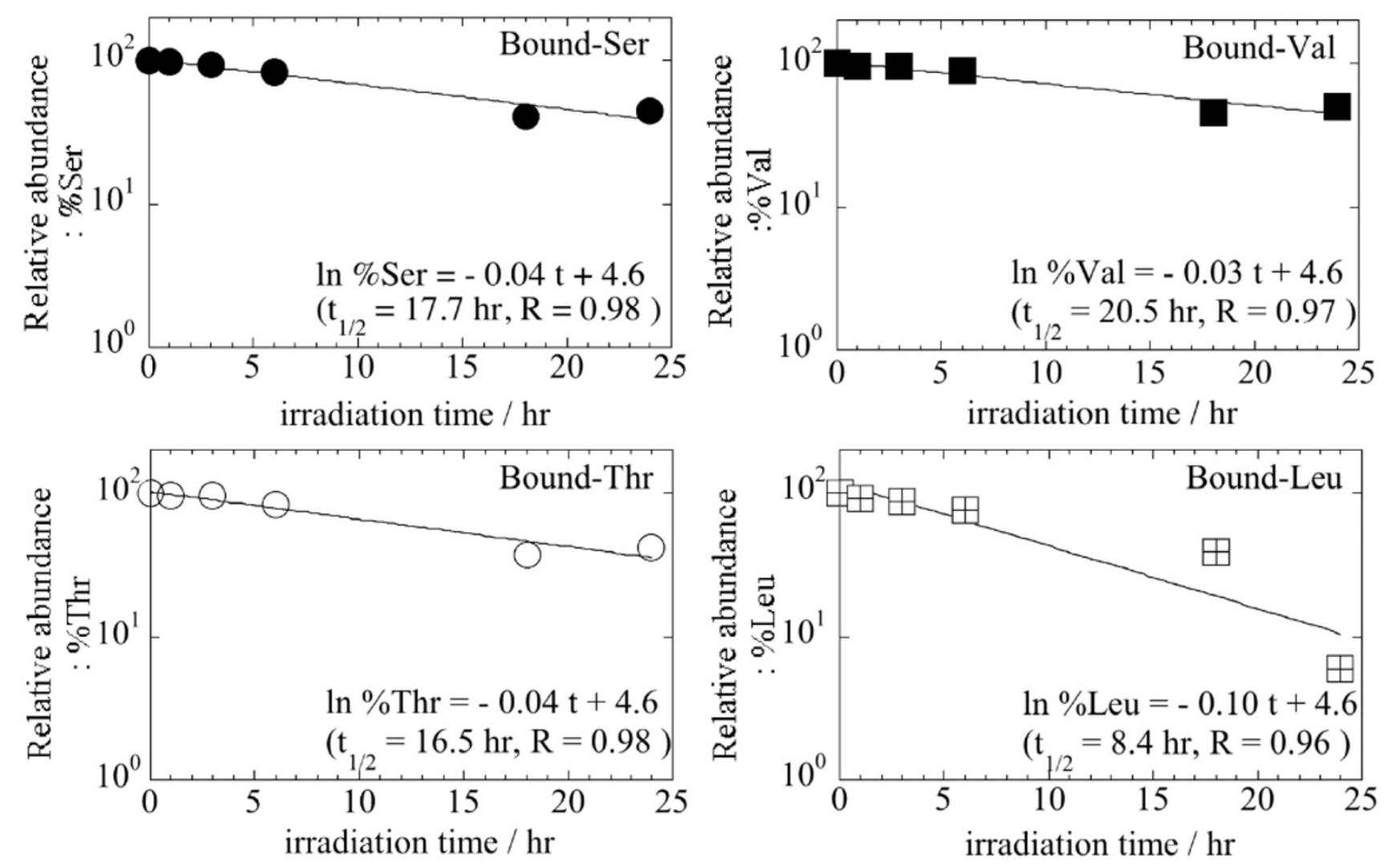

Fig. 2(b). Semi-logarithmic linear relations between relative abundance and radiation time to determine half-life of bound amino acid analogs by $\gamma$-rays irradiation experiment. Bound amino acids of serine, valine, threonine and leucine were shown as typical examples. 
Table 1. The comparison of stability of free- and bound-amino acids by $\gamma$-rays and UV irradiation experiment.

\begin{tabular}{|c|c|c|c|c|c|c|c|}
\hline \multirow[b]{3}{*}{ Amino acids } & \multicolumn{7}{|c|}{-rays irradiation experiment } \\
\hline & \multicolumn{3}{|c|}{ Free amino acids } & \multicolumn{3}{|c|}{ Bound amino acids } & \multirow[t]{2}{*}{ Bound/Free } \\
\hline & slope & $t \quad 1 / 2$ & $J 1 / 2$ & slope & $t 1 / 2$ & $J_{1 / 2}$ & \\
\hline Asp & -0.19 & 6.2 & 44.2 & -0.02 & 46.1 & 329.3 & 7.5 \\
\hline Thr & -0.67 & 1.8 & 13.1 & -0.04 & 16.5 & 118.2 & 9.0 \\
\hline Ser & -0.60 & 1.7 & 11.9 & -0.04 & 17.7 & 126.4 & 10.6 \\
\hline Glu & -0.25 & 0.9 & 6.3 & -0.03 & 24.6 & 176.1 & 27.9 \\
\hline -AAA & -0.73 & 1.2 & 8.4 & - & - & - & n.d. \\
\hline Ala & -0.12 & 7.4 & 52.8 & -0.03 & 27.1 & 193.7 & 3.7 \\
\hline$-\mathrm{ABA}$ & -0.69 & 1.9 & 13.5 & - & - & - & n.d. \\
\hline Val & -0.74 & 1.2 & 8.4 & -0.03 & 20.5 & 146.9 & 17.5 \\
\hline Ile & -4.96 & 0.6 & 4.5 & -0.08 & 9.2 & 65.5 & 14.5 \\
\hline Leu & -1.42 & 0.7 & 4.8 & -0.10 & 8.5 & 60.4 & 12.6 \\
\hline Tyr & -2.08 & 0.3 & 2.4 & -0.11 & 3.2 & 22.8 & 9.6 \\
\hline Phe & -4.02 & 0.2 & 1.2 & -0.06 & 8.7 & 62.4 & 50.6 \\
\hline -Ala & -0.21 & 4.7 & 33.9 & - & - & - & n.d. \\
\hline -AiBA & -0.51 & 2.0 & 14.2 & - & - & - & n.d. \\
\hline
\end{tabular}

\begin{tabular}{|c|c|c|c|c|c|c|c|}
\hline \multirow[b]{3}{*}{ Amino acids } & \multicolumn{7}{|c|}{ UV irradiation experiment } \\
\hline & \multicolumn{3}{|c|}{ Free amino acids } & \multicolumn{3}{|c|}{ Bound amino acids } & \multirow[t]{2}{*}{ Bound / Free } \\
\hline & slope & $t_{1 / 2}$ & $J 1 / 2$ & slope & $t 1 / 2$ & $J_{1 / 2}$ & \\
\hline Asp & -0.03 & 24.8 & 50.8 & -0.01 & 67.0 & 137.3 & 2.7 \\
\hline Thr & -0.20 & 5.1 & 10.4 & -0.02 & 32.1 & 65.8 & 6.3 \\
\hline Ser & -0.21 & 3.9 & 8.1 & -0.02 & 49.8 & 102.1 & 12.7 \\
\hline Glu & -0.24 & 2.9 & 5.9 & -0.02 & 41.0 & 84.1 & 14.3 \\
\hline -AAA & -0.27 & 2.4 & 4.9 & - & - & - & n.d. \\
\hline Ala & -0.09 & 9.0 & 18.4 & -0.01 & 75.7 & 155.2 & 8.4 \\
\hline$-\mathrm{ABA}$ & -0.25 & 3.5 & 7.3 & - & - & - & n.d. \\
\hline Val & -0.34 & 2.6 & 5.3 & -0.02 & 36.1 & 74.1 & 14.0 \\
\hline Ile & -0.38 & 1.8 & 3.7 & -0.03 & 28.2 & 57.8 & 15.5 \\
\hline Leu & -0.38 & 1.9 & 3.8 & -0.02 & 30.5 & 62.6 & 16.5 \\
\hline Tyr & -1.84 & 0.3 & 0.7 & - & - & - & n.d. \\
\hline Phe & -1.43 & 0.5 & 1.1 & -0.05 & 14.7 & 30.2 & 28.2 \\
\hline -Ala & -0.07 & 10.0 & 20.5 & - & - & - & n.d. \\
\hline -AiBA & -0.20 & 3.9 & 8.0 & - & - & - & n.d. \\
\hline
\end{tabular}

Each slope and half-life $(t \quad 1 / 2)$ were determined by semi-logarithmic relationship.

The stability of amino acids were compared with the ratio of bound analogs per free analogs.

$J 1 / 2$ stands for half of corresponding energy deposit. n.d. is not determined.

and results, Section 3.1). Following irradiation, an aliquot of the irradiation products were hydrolyzed with $6 \mathrm{~mol} \cdot \mathrm{dm}^{-3}$ $\mathrm{HCl}$ at $110^{\circ} \mathrm{C}$ for 24 hours, then evaporated to dryness, and adjusted to $\mathrm{pH} 1$ (Takano et al., 2001). Amino acids in the hydrolyzed fraction were analyzed by ion-exchange high performance liquid chromatograph (HPLC) system using post-column derivatization with $o$-phthalaldehyde and $\mathrm{N}$ acetyl-L-cystein. The HPLC system was composed of two HPLC pumps (Shimadzu LC-6A), a cation exchange column (4 mm i.d. $150 \mathrm{~mm}$; Shimpak ISC-07/S1504), a post column derivatization system, and a Shimadzu RF-535 fluorometric detector (excited wavelength, $355 \mathrm{~nm}$ and emission wavelength, $435 \mathrm{~nm}$ ). The temperature of the column was maintained at $55^{\circ} \mathrm{C}$. Gradient elution was performed using eluents $\mathrm{A}(0.07 \mathrm{M}$ sodium citrate perchloric acid, $\mathrm{pH} 3.20$ with $7 \%$ ethanol) and $\mathrm{B}(0.2 \mathrm{M}$ sodium citrate boric acid- $\mathrm{NaOH}$,
$\mathrm{pH}$ 10). The flow rate of the carrier was $0.3 \mathrm{ml} / \mathrm{min}$. The data reproducibility in the present procedure are better than $\pm 5 \%$ for relative molar concentrations of the amino acids. Prior to use, all glass ware was heated at $500^{\circ} \mathrm{C}$ in a high temperature oven (Yamato DR-22) to eliminate any possible contaminants.

\section{Result and Discussion}

The stability of free and bound amino acids. Typical ion-exchange chromatograms of free amino acids following $\gamma$-rays irradiation are shown in Fig. 1. The concentrations of free amino acids drastically decreased with increasing duration of irradiation. However, apparent concentrations of bound amino acids in the protein solutions decreased only slightly during the irradiation experiments. The semi-logarithmic linear relationship found between the rela- 

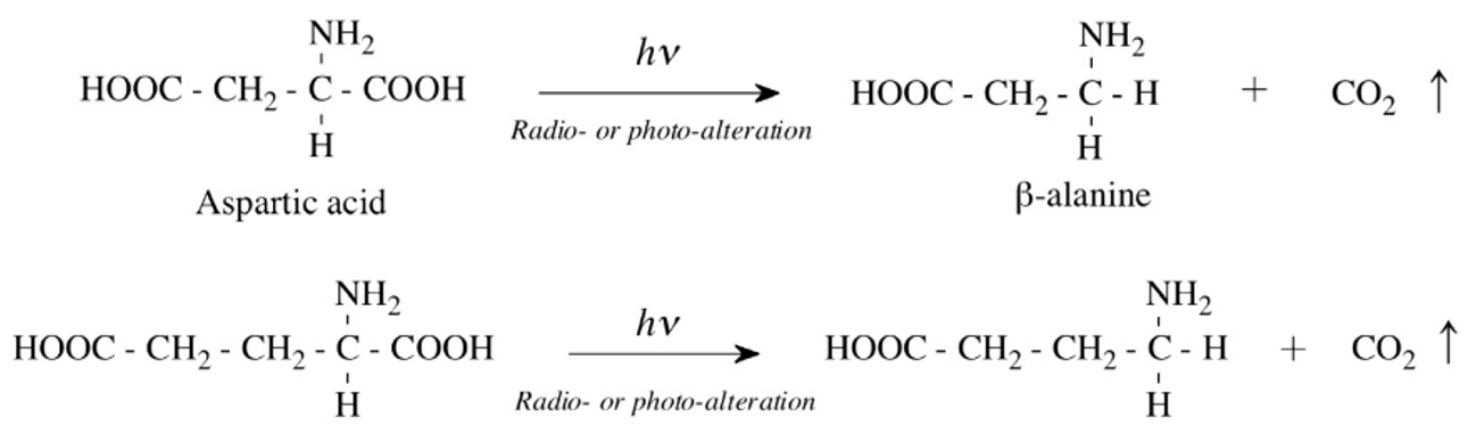

Glutamic acid

$\gamma$-aminobutyric acid

Fig. 3. Possible pathway of formation of $\beta$-alanine and $\gamma$-aminobutyric acid from aspartic acid and glutamic acid, respectively, as secondary amino acids by $\gamma$-rays and UV irradiation. This process was observed from both fraction of free and bound amino acid analogs.

tive abundance of amino acids and irradiation time is shown in Fig. 2. As shown in Figs. 2(a) and 2(b), the bound amino acids are more stable than the free analogs; for example, the half-lives $\left(t_{1 / 2}\right)$ of bound serine and threonine under $\gamma$-rays irradiation are more than 10.6 and 27.9 times longer, respectively, than the free analogs. The half-lives $\left(t_{1 / 2}\right)$ of bound valine and leucine in $\gamma$-rays irradiation are more than 17.5 and 12.6 times longer, respectively, than that of the frees. Other amino acids for which the bound analogs was generally more stable than that of the free analogs are shown in Table 1. Tyrosine and phenylalanine with aromatic constituents are unstable in the monomer soluble condition, but are far more stable during irradiation as bound analogs. This quantitatively shows that bound analogs are much more stable than free analogs when subjected to $\gamma$-rays and UV irradiation. The present data clearly carries significant implications for the fate of amino acids under irradiation in asteroids and comets; free amino acids hardly survive, while bound amino acids, including amino acid precursors remain intact.

Secondary products of $\beta$-alanine and $\gamma$-aminobutyric acid. $\quad \gamma$-aminobutyric acid were detected in free and bound analogs during irradiation (Fig. 1). In another irradiation experiment using $\gamma$-rays and UV irradiation on aspartic acid and glutamic acid solutions, $\beta$-alanine and $\gamma$ aminobutyric acid, respectively, were produced. It has been reported that aspartic acid and glutamic acid may undergo $\alpha$-decarboxylation by a pyrolysis reaction, that is, glutamic acid is altered to $\gamma$-aminobutyric acid by decarboxylation at the $\alpha$-carbon (Ratcliff et al., 1974). In terrestrial organic sediments, similar observations are made in the early stages of natural sedimentary alteration or diagenesis (Cowie and Hedges, 1994; Takano et al., 2004a). Consequently, secondary amino acids $\beta$-alanine and $\gamma$-aminobutyric acid might be formed by radio- or photo-decomposition processes from aspartic acid and glutamic acid, respectively (Fig. 3).

In the present paper, we used mixtures of multiple amino acids. However, because irradiated amino acids may be altered to form glycine by radio- or photo-decomposition processes and methionine is known to undergo hydrolytic loss during acid hydrolysis (Andersson et al., 2000; Takano et al., 2004a, 2004b), these amino acids were excluded from the quantitative evaluation in Table 1.

Detection of extraterrestrial amino acids by telescopic method. In the search for extraterrestrial amino acids, the simplest amino acid, glycine, has been the target of telescopic observation in the interstellar medium (Snyder, 1997). Because glycine plays a key role in the formation of proteins and other biological molecules, it is worth resuming this research, which has been largely unsuccessful until recently. The possible presence of glycine in molecular clouds was reported (Snyder, 1997), but the signal has not been confirmed yet (Ohishi, 2000). Recently, successful detection of possible interstellar glycine was reported by Kuan et al. (2003, 2004). Hence, the discovery of interstellar glycine may strengthen the exogenous study that interstellar molecules could have played pioneering role in the prebiotic chemistry of the early Earth.

Thus the difficulty in finding glycine might be consistent with the present results showing that intact monomer amino acid analogs are rare in extraterrestrial environments, while bound amino acid precursors are easily detected in the analysis of meteorites (Cronin et al., 1988) and produced in laboratory simulation experiments (Kobayashi et al., 1995, 1998; Kasamatsu et al., 1997). As to simulation experiment of proton irradiation which is main component of cosmic rays, Takano et al. (2004c) experimentally verified the abiotic formation of high-molecular-weight organics from an interstellar medium gas mixture of carbon monoxide, ammonia, and water as a result of $3-\mathrm{MeV}$ proton irradiation from a Van de Graaff accelerator. Then the irradiation products included amino acid precursors and the molecular weight distribution ranged from several hundred to a maximum of 3,000 identified by Gel Filtration High Performance Liquid Chromatography (HPLC) system. The primary irradiation products were proved to be not free amino acid analogs but were bound amino acid precursors having high-molecular-weight. In addition, high-molecular-weight organic matter synthesized from gas mixtures of carbon monoxide, ammonia and water vapor were irradiated with a $3 \mathrm{MeV}$ proton beam in order to analyze by Curie-point pyrolysis with detection by a gas chromatograph and a mass spectrometer (Pyr-GC-MS) (Takano et al., 2004d). Pyr-GC-MS experiment gave a wide variety of organic compounds, not only a number of amide compounds, but also heterocyclic and polycyclic aromatic hydrocarbons (PAHs) among the products of the pyrolysis (Takano et al., 2004d). Detection of biologically interesting compounds, such as glycolamide $\left(\mathrm{HOCH}_{2} \mathrm{CONH}_{2}\right)$, is also cosmochemically interesting. The pyrolysis experimen- 
tal data also shows that primary and primitive organic matter serving as "bound precursors" to bioorganic compounds, such as amino acids, nucleic acid bases, and sugar, might have been formed in a gaseous mixture of similar composition to that of the interstellar dust environment (Takano et $a l ., 2004 \mathrm{~d})$. Consequently, the delivery of exogenous organic carbon containing bound amino acid precursors by comets and meteorites may have played an important role in the early stages of chemical evolution on the Earth (Cottin et al., 1999).

\section{Conclusions}

The following characteristics with regard to photostability of free and bound amino acids by $\gamma$-rays and UV irradiation experiments were found:

1) Increasing duration of $\gamma$-rays and UV irradiation drastically decreased free amino acid analog and slightly decreased bound amino acid analog concentrations. Hence, the photo-stability of bound amino acid analogs was greater than that of free amino acid analogs under high-energy photons.

2) Radio- or photo-alteration processes produce secondary amino acid products via decomposition of the $\alpha$-carboxylic group. Aspartic acid and glutamic acids are converted to $\beta$ alanine and $\gamma$-aminobutyric acids by $\gamma$-rays and UV irradiation, and $\alpha$-decarboxylation photolysis of these dicarboxylic amino acid were observed.

Acknowledgments. The authors express their sincere thanks to Dr. Max P. Bernstein, Ames Research Center of National Aeronautics \& Space Administration (NASA) and Dr. Hirofumi Hashimoto, Institute of Engineering Mechanics \& Systems, University of Tsukuba for their critical and constructive reviewing comments which helped to improve the earlier version of the manuscript. The authors also thank to associate editor Dr. Sho Sasaki, Department of Earth \& Planetary Science, University of Tokyo for the manuscript handling and suitable suggestion. This research was supported in part by a Grant-In-Aid (No. 14340170) from MEXT (Ministry of Education, Culture, Sports, Science and Technology, Japan) and the Special Co-ordination Fund for the Archaean Park Project.

\section{References}

Andersson, E., B. R. T. Simoneit, and N. G. Holm, Amino acid abundance and stereochemistry in hydrothermally altered sediments from the Juan de Fuca Ridge, northeastern Pacific Ocean, Appl. Geochem., 15, 11691190,2000

Bernstein, M. P., J. P. Dworkin, S. A. Sandford, G. W. Cooper, and L. J. Allamandora, Racemic amino acids from the ultraviolet photolysis of interstellar ice analogues, Nature, 416, 401-403, 2002.

Briggs, R., G. Ertem, J. P. Ferris, J. M. Greenberg, P. J. Mcain, C. X. Mendoza-Gomes, and W. Shutte, Comet Halley as an aggregate of interstellar dust and further evidence for the photochemical formation of organics in the interstellar medium, Origins Life Evol. Biosphere, 22, 287-307, 1992.

Cowie, G. L. and J. I. Hedges, Biochemical indicators of diagenetic alteration in natural organic matter mixtures, Nature, 369, 304-307, 1994.

Cottin, H., M. C. Gazeau, and F. Raulin, Cometary organic chemistry: a review from observations, numerical and experimental simulations, Planet. Space Sci., 47, 1141-1162 1999.

Cronin, J. R., S. Pizzarello, and D. P. Cruikshank, "Meteorites and the Early Solar System", edited by J. F. Kerridge and M. S. Matthews, The University of Arizona Press (1988), pp. 819-857.

Cronin, J. R. and S. Pizzarello, Enantiomeric excesses of meteoritic amino acids, Science, 275, 951-955, 1997.

Ehrenfreund, P. and S. B. Charnley, Organic molecules in the interstellar medium, comets, and meteorite: A voyage from dark clouds to the early Earth, Annu. Rev. Astron. Astrophys, 38, 427-483, 2000.

Ehrenfreund, P., M. P. Bernstein, J. P. Dworkin, S. A. Sandford, and L. J.
Allamandola, The photostability of amino acids in space, Astrophys. $J$ Lett., 550, L95-L99, 2001.

Ikeda, H. and A. Suzuki, Radiolysis of $n$-dodecane and its physical property change based on the dose in one pass through a reference HA column, $J$. Nuclear Sci. Tech., 35, 697-704, 1998

Kasamatsu, T., T. Kaneko, T. Saito, and K. Kobayashi, Formation of organic compounds in simulated interstellar media with high-energy particles, Bull. Chem. Soc. Jpn., 70, 1021-1026, 1997.

Kobayashi, K. and T. Tsuji, Abiotic synthesis of uracil from carbon monoxide, nitrogen and water by proton irradiation, Chem. Lett., 903-904, 1997. Kobayashi, K., T. Kasamatsu, T. Kaneko, J. Koike, T. Oshima, T. Saito, T. Yamamoto, and H. Yanagawa, Formation of amino acid precursors in cometary ice environments by cosmic radiation, Adv. Space Res., 16, 21 26, 1995.

Kobayashi, K., T. Kaneko, T. Saito, and T. Oshima, Amino acids formation in gas mixtures by high-energy particle irradiation, Origins Life Evol. Biosphere, 28, 155-165, 1998.

Kobayashi, K., H. Masuda, K. Ushio, A. Ohashi, H. Yamanashi, T. Kaneko, J. Takahashi, T. Hosokawa, H. Hashimoto, and T. Saito, Formation of bioorganic compounds in simulated planetary atmospheres by high energy particles or photon, Adv. Space Res., 27, 207-215, 2001.

Kobayashi, K., Y. Takano, H. Masuda, H. Tonishi, T. Kaneko, H. Hashimoto, and T. Saito, Possible cometary organic compounds as sources of planetary biospheres, Adv. Space. Res., 33, 1277-1281, 2004.

Kuan, Y.-J., S. B. Charnley, H. Hui-Chun, T. Wei-Ling, and K. Zbigniew, Interstellar glycine, Astrophys. J., 593, 848-867, 2003.

Kuan, Y.-J., S. B. Charnley, H. Hui-Chun, T. Wei-Ling, K. Zbigniew, P. Ehrenfreund, T. Wei-Ling, and Y. Chi-Hung, Searches for interstellar molecules of potential prebiotic importance, Adv. Space Res., 33, 31-39, 2004.

Miller, S. L, The production of amino acids under possible primitive earth conditions, Science, 117, 528-531, 1953.

Miyakawa, S., H. Yamanashi, K. Kobayashi, H. J. Cleaves, and S. L. Miller, Prebiotic synthesis from CO atmospheres: Implications for the origins of life, Proc. Nat. Acad. Sci. USA, 99, 14628-14631, 2002.

Munoz Caro, G. M., U. J. Meierhenrich, W. A. Schutte, B. Barbier, A. A. Segavia, H. Rosenbauer, W. H. P. Thiemann, A. Brack, and J. M. Greenberg, Nature, 416, 403-405, 2002.

Ohishi, M., Disscussion on the relationship between interstellar matter and molecules for origin of life, Viva Origino, 28, 175-190, 2000.

Pizzarello, S. and J. R. Cronin, Non-racemic amino acids in the Murray and Murchison meteorites, Geochim. Cosmochim. Acta, 64, 329-338, 2000.

Ratcliff, M. A., E. E. Medeley, and P. G. Simmonds, Pyrolysis of amino acids. mechanistic considerations, J. Org. Chem., 39, 1481-1490, 1974.

Synder, L. E., The search for interstellar glycine, Origins Life Evol. Biosphere, 27, 115-133, 1997.

Takano, Y., K. Ushio, H. Masuda, T. Kaneko, K. Kobayashi, J. Takahashi, and T. Saito, Determination of organic compounds formed in simulated interstellar dust environment, Anal. Sci., 17, 1635-1648, 2001.

Takano, Y., H. Masuda, T. Kaneko, and K. Kobayashi, Formation of amino acids from possible interstellar media by $\gamma$-rays and UV irradiation, Chem. Lett., 10, 986-987, 2002.

Takano, Y., K. Ushio, T. Kaneko, K. Kobayashi, and H. Hashimoto, Amino acid precursors from carbon monoxide in simulated interstellar dust ice mantle by UV irradiation at $10 \mathrm{~K}$, Chem. Lett., 2, 612-613, 2003.

Takano, Y., J. Kudo, T. Kaneko, K. Kobayashi, Y. Kawasaki, and Y. Ishikawa, Distribution of amino acids and its stereo chemistry related with biological activities in Rikubetsu, Hokkaido, Japan. Geochem. J., 38, 153-161, 2004a.

Takano, Y., T. Kaneko, K. Kobayashi, and K. Marumo, Correlation coefficients between biomarkers and sub-surface microbial activities in terrestrial sediment over the past 10,000 years, Bunseki Kagaku, 53, 167-172, 2004b (in Japanese with English abstract).

Takano, Y., A. Ohashi, T. Kaneko, and K. Kobayashi, Abiotic synthesis of high-molecular-weight organics containing amino acid precursors from inorganic gas mixture of carbon monoxide, ammonia and water by $3 \mathrm{MeV}$ proton irradiation, Appl. Phys. Lett., 84, 1410-1412, $2004 \mathrm{c}$.

Takano, Y., T. Tsuboi, T. Kaneko, K. Kobayashi, and K. Marumo, Pyrolysis of high-molecular-weight complex organics synthesized from simulated interstellar gas mixture irradiated with $3 \mathrm{MeV}$ proton irradiation, Bull. Chem. Soc. Jpn., 77, 779-783, 2004d.

Y. Takano (e-mail: takano.yoshinori@aist.go.jp), T. Kaneko, K. Kobayashi, D. Hiroishi, H. Ikeda, and K. Marumo 